\title{
Two new methods for removing salt-and-pepper noise from digital images
}

\author{
Uğur Erkan ${ }^{\mathrm{a}}$, Adem Kilicman ${ }^{\mathrm{b}, *}$ \\ a Erbaa Vocational School, Gaziosmanpaşa, 60500 Erbaa, Tokat, Turkey \\ b Department of Mathematics and Institute for Mathematical Research, University Putra Malaysia, \\ 43400 UPM, Serdang, Selangor, Malaysia
}

*Corresponding author, e-mail: akilic@upm.edu.my

Received 31 Oct 2014

Accepted 14 Dec 2015

\begin{abstract}
In this study, we developed two new alternative methods to enhance images which operate satisfactorily with high levels of noise in any given window size. We also conducted a test by adding the most frequently used noise type to the test images of salt-and-pepper noise. The results are compared with other well-known noise remover methods in the literature such as, standard median filter (SMF) and adaptive median filter (AMF), and much better results were obtained. For instance, the results of SMF and AMF filters as well as peak signal-to-noise ratio (PSNR) are 33.2 and 29.8 respectively by adding a ratio of 0.1 salt-and-pepper noise to the Lena image. With the new methods, the results are 41.4 and 41.4. The newly developed methods also generate better results in reducing high volume of noise. For instance, if a ratio of 0.5 salt-and-pepper noise is applied to a Lena image, PSNR results are 15.22 and 22.78 in SMF and AMF, and 25.8 and 28.9 with newly developed methods.
\end{abstract}

KEYWORDS: image processing, peak signal-to-noise ratio, image enhancement factor, elastic median filters

\section{INTRODUCTION}

Salt-and-pepper noise was introduced to the images to test both methods. We checked the quality of the filters by adding salt-and-pepper noise of 0.1-0.9. The first filter in which the distances are controlled by a coefficient is, therefore, assumed to remove any other noise. Salt-and-pepper noise is commonly removed using a standard median filter (SMF). In removal of salt-and-pepper noise, better results have been obtained in square matrix window sizes where SMF generates poor results (unlike $3 \times 3,5 \times 5$ ) . The underlying reason for developing these algorithms was our foresight to provide flexibility for SMF algorithm.

Salt-and-pepper noise adjusting pixel values of the image as the lowest and highest pixel values (0 and 255, for 8 bits) is randomly applied to pixel values to an image depending upon the level of percentage. A variety of methods are available for the removal of this type of noise, and SMF is the most widely used method.

Adaptive median filter (AMF) developed from SMF provides better results than SMF. This method, however, makes an image more blurry, thus making the edges to disappear. Many alternative median filters are available to provide solution to this problem. One of the methods offering a solution is the decision based algorithm (DBA), a sorting algorithm. It works with a variational method preserving the fine details in noise removal ${ }^{1}$. The following two filters operating just non-defective pixels and avoiding defective ones were developed: decision based unsymmetric trimmed median filter (DBUTMF) and modified decision based unsymmetric trimmed median filter (MDBUTMF) ${ }^{2,3}$. Another method is BDND where defective pixels are removed with an EXTERNAL coefficient and a directional vector is applied to median filter to remove noise ${ }^{4}$. Local classification of the pixels prior to noise removal is another method ${ }^{5}$. A filter using histogram to pinpoint a noise was developed as an alternative to median filter ${ }^{6}$. Fuzzy set theory and many others have recently been developed. The method known as fuzzy-based decision algorithm in literature (FBDA) was strong in preserving the details but weak in preserving the edges in high levels of noise ${ }^{7-11}$.

In the evaluation of the developed methods, peak signal-to-noise ratio (PSNR) and image enhancement factor (IEF) were applied. PSNR is calculated by determining the pixel discrepancy between the base picture and the processed picture.

PSNR is defined by mean squared error (MSE). 
MSE is computed from

$$
\mathrm{MSE}=\frac{1}{m n} \sum_{i=0}^{m-1} \sum_{j=0}^{n-1}[I(i, j)-K(i, j)]^{2}
$$

where $I, m \times n$ is the noiseless image and $K$ is the noisy image. The PSNR is given by

$$
\mathrm{PSNR}=10 \log _{10}\left(\frac{\mathrm{MAX}_{I}^{2}}{\mathrm{MSE}}\right),
$$

here, $\mathrm{MAX}_{I}$ is the maximum possible pixel value of the image. When the pixels are represented using 8 bits per sample, this is $255^{12}$.

Image enhancement factor (IEF) is defined as

$$
\mathrm{IEF}=\frac{\sum_{i=1}^{M} \sum_{j=1}^{N}[x(i, j)-I(i, j)]^{2}}{\sum_{i=1}^{M} \sum_{j=1}^{N}[K(i, j)-I(i, j)]^{2}} .
$$

In these equations, the size of the original image $I$ is $M \times N$, and the reconstructed image is $K$. Moreover, the noisy pixel is denoted by $x^{13,14}$.

This study aims to develop two alternative methods for noise removal from images.

\section{NEW METHODS}

The shortcomings of SMF are as follows.

(i) Poor performance in working with high levels of noise (satisfactory performance in lower levels, $0.1)$.

(ii) Better performance given the square matrix $(3 \times 3,5 \times 5)$ and unsatisfactory performance in other windows sizes.

(iii) Flexibility for only the given parameters.

The two filters we are going to develop are aimed to operate satisfactorily not only with high levels of noise but also in any given window size. The filter is aimed to gain flexibility by adding a value of parameter to the first algorithm.

Many methods operate on defective filters (0,256 in 8-bit images) avoiding non-defective ones in removing salt-and-pepper noise. We obtained better results by controlling all the pixels by means of a range. Additionally, the developed filter generates better results in not only salt-and-pepper noise but also with other types of noise such as Gaussian and speckle.

The developed methods are to be named as 'elastic median filter 1' (EMF1) and 'elastic median filter 2' (EMF2).

\section{Steps of the algorithm 1 (EMF1)}

This algorithm was developed based on calculating the difference between pixels, and addition of

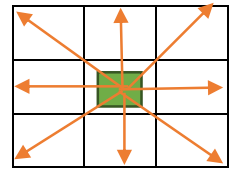

\begin{tabular}{|c|c|c|}
\hline 159 & 155 & 255 \\
\hline 157 & 153 & 159 \\
\hline 255 & 255 & 0 \\
\hline
\end{tabular}

Fig. 1 Filter window for $3 \times 3$.

\begin{tabular}{|c|c|c|}
\hline 0 & 153 & 155 \\
\hline 157 & 159 & 159 \\
\hline 255 & 255 & 255 \\
\hline
\end{tabular}

Fig. 2 Sorted array.

a coefficient to SMF. In the algorithm, a new array is created, and the difference between the middle value and other array elements is found. The working principle of this algorithm is based on the comparison of processed pixel where processed value of the parameter is either added to or removed from this value.

The steps of the algorithm are as follows:

Step 1: An array is created according to a given window size. Fig. 1 provides an example for a $3 \times 3$ array. The pixel in the middle operates as a matrix

$$
\text { A1 }=(159,155,255,157,153,159,255,255,0) \text {. }
$$

Step 2: Pixels are sorted from lowest to highest.

$$
\mathrm{A} 2=(0,153,155,157,159,159,255,255,255) \text {. }
$$

Step 3: A filter variable is created with a given coefficient. When the window size is $[m n]$ and the coefficient is $\beta$, then

$$
\alpha=(m n)^{\beta} .
$$

For instance, when $\beta=1.8$, for Fig. 1,

$$
\alpha=((3)(3))^{1.8} \approx 52 .
$$

Step 4: The difference between middle values and other values in the array in Step 2 is calculated.

If a distance is $\sigma$ (sum of the absolute differences between each pixel and the central pixel),

$$
\begin{array}{r}
\sigma=(159-0)+(159-153)+(159-155)+(159- \\
157)+(255-159)+(255-159)+(255-159),
\end{array}
$$

Step 5: Let processed pixel value be $P_{\mathrm{e}}$ (153 for Fig. 1), and $P_{\mathrm{y}}$ for middle pixel value in Fig. 2, we obtain:

$$
\text { If } P_{\mathrm{e}} \geqslant P_{\mathrm{y}}+\alpha+\sqrt{\sigma} \text { or } P_{\mathrm{e}} \leqslant P_{\mathrm{y}}-\alpha-\sqrt{\sigma},
$$

then

$$
P_{\mathrm{e}}=P_{\mathrm{y}}
$$


Table 1 PSNR and IEF results.

\begin{tabular}{|c|c|c|c|c|c|c|c|c|c|c|c|c|c|c|c|c|c|}
\hline \multicolumn{9}{|c|}{ Lena } & \multicolumn{9}{|c|}{ Peppers } \\
\hline \multirow[t]{2}{*}{ NR } & \multicolumn{4}{|c|}{ PSNR } & \multicolumn{4}{|c|}{ IEF } & \multirow[t]{2}{*}{$\overline{\mathrm{NR}}$} & \multicolumn{4}{|c|}{ PSNR } & \multicolumn{4}{|c|}{ IEF } \\
\hline & SMF & AMF & EMF1 & EMF2 & SMF & AMF & EMF1 & EMF2 & & SMF & AMF & EMF1 & EMF2 & SMF & AMF & EMF1 & EMF2 \\
\hline 0.1 & 33.2 & 29.8 & 41.4 & 41.4 & 59.8 & 26.9 & 394.7 & 392.9 & 0.1 & 31.7 & 29.6 & 40.1 & 40.3 & 43.3 & 26.7 & 301.1 & 316.1 \\
\hline 0.2 & 29.1 & 27.1 & 37.4 & 37.1 & 46.3 & 29.2 & 312.9 & 291.4 & 0.2 & 28.0 & 26.8 & 36.1 & 36.7 & 37.1 & 28.2 & 241.6 & 275.3 \\
\hline 0.3 & 23.6 & 25.2 & 33.2 & 33.9 & 19.3 & 28.4 & 178.1 & 209.8 & 0.3 & 23.3 & 25.0 & 33.3 & 34.2 & 18.8 & 27.9 & 184.9 & 227.9 \\
\hline 0.4 & 18.9 & 23.8 & 29.4 & 31.1 & 8.9 & 27.3 & 99.5 & 146.4 & 0.4 & 18.7 & 23.6 & 29.7 & 31.5 & 8.7 & 27.2 & 109.6 & 166.4 \\
\hline 0.5 & 15.2 & 22.8 & 25.8 & 28.9 & 4.7 & 26.9 & 54.0 & 110.8 & 0.5 & 15.1 & 22.6 & 25.6 & 29.2 & 4.8 & 26.9 & 53.7 & 123.1 \\
\hline 0.6 & 12.4 & 21.8 & 21.9 & 26.7 & 2.9 & 25.7 & 26.4 & 80.1 & 0.6 & 12.2 & 21.7 & 21.7 & 26.8 & 2.9 & 26.1 & 26.3 & 84.1 \\
\hline 0.7 & 10.0 & 20.9 & 18.2 & 24.5 & 2.0 & 24.1 & 13.1 & 55.6 & 0.7 & 9.9 & 20.6 & 17.8 & 24.4 & 2.0 & 23.5 & 12.5 & 57.1 \\
\hline 0.8 & 8.2 & 17.7 & 14.3 & 20.9 & 1.5 & 13.5 & 6.1 & 27.9 & 0.8 & 8.1 & 17.9 & 14.4 & 20.6 & 1.5 & 14.4 & 6.4 & 26.9 \\
\hline 0.9 & 6.7 & 12.3 & 10.3 & 16.4 & 1.2 & 4.4 & 2.7 & 11.1 & 0.9 & 6.6 & 12.2 & 10.3 & 14.9 & 1.2 & 4.4 & 2.8 & 8.2 \\
\hline \multicolumn{9}{|c|}{ Cameraman } & \multicolumn{9}{|c|}{ Plane } \\
\hline \multirow[t]{2}{*}{$\overline{\mathrm{NR}}$} & \multicolumn{4}{|c|}{ PSNR } & \multicolumn{4}{|c|}{ IEF } & NR & \multicolumn{4}{|c|}{ PSNR } & \multicolumn{4}{|c|}{ IEF } \\
\hline & SMF & $\mathrm{AMF}$ & EMF1 & EMF2 & SMF & $\mathrm{AMF}$ & EMF1 & EMF2 & & SMF & AMF & EMF1 & EMF2 & SMF & AMF & EMF1 & EMF2 \\
\hline 0.1 & 33.7 & 30.1 & 41.6 & 41.0 & 71.6 & 31.6 & 445.6 & 384.4 & 0.1 & 31.7 & 31.7 & 40.5 & 40.7 & 47.0 & 47.0 & 358.3 & 379.6 \\
\hline 0.2 & 28.6 & 27.2 & 36.9 & 36.6 & 44.9 & 32.5 & 301.4 & 279.4 & 0.2 & 27.6 & 27.6 & 36.1 & 36. & 36.5 & 36.5 & 257.4 & 306.3 \\
\hline 0.3 & 23.3 & 25.5 & 33.4 & 33.5 & 19.8 & 33.0 & 200.8 & 206.5 & 0.3 & 22.9 & 22.9 & 32.9 & 33.7 & 18.6 & 18.6 & 183.7 & 225.2 \\
\hline 0.4 & 18.6 & 24.0 & 29.4 & 30.7 & 8.9 & 30.8 & 106.4 & 143.1 & 0.4 & 18.2 & 18.2 & 28.8 & 31.4 & 8.5 & 8.5 & 97.3 & 176.7 \\
\hline 0.5 & 14.9 & 22.9 & 25.1 & 28.0 & 4.8 & 29.7 & 50.2 & 96.3 & 0.5 & 14.6 & 14.6 & 25.2 & 28.9 & 4.6 & 4.6 & 52.3 & 125.5 \\
\hline 0.6 & 12.0 & 21.8 & 21.6 & 25.5 & 2.9 & 27.8 & 26.7 & 65.9 & 0.6 & 11.8 & 11.8 & 21.2 & 26.6 & 2.9 & 2.9 & 25.1 & 87.8 \\
\hline 0.7 & 9.7 & 20.7 & 17.7 & 22.7 & 2.0 & 25.1 & 12.8 & 40.3 & 0.7 & 9.5 & 9.5 & 17.5 & 24.4 & 2.0 & 2.0 & 12.6 & 62.0 \\
\hline 0.8 & 7.9 & 17.7 & 14.1 & 19.8 & 1.5 & 14.3 & 6.2 & 23.7 & 0.8 & 7.6 & 7.6 & 13.9 & 21.4 & 1.5 & 1.5 & 6.2 & 35.1 \\
\hline 0.9 & 6.3 & 11.9 & 9.9 & 15.9 & 1.2 & 4.3 & 2.7 & 10.7 & 0.9 & 6.1 & 6.1 & 9.8 & 18.0 & 1.2 & 1.2 & 2.7 & 18.0 \\
\hline \multicolumn{9}{|c|}{ Baboon } & \multicolumn{9}{|c|}{ Bridge } \\
\hline \multirow[t]{2}{*}{ NR } & \multicolumn{4}{|c|}{ PSNR } & \multicolumn{4}{|c|}{ IEF } & NR & \multicolumn{4}{|c|}{ PSNR } & \multicolumn{4}{|c|}{ IEF } \\
\hline & SMF & AMF & EMF1 & EMF2 & SMF & AMF & EMF1 & EMF2 & & SMF & AMF & EMF1 & EMF2 & SMF & AMF & EMF1 & EMF2 \\
\hline 0.1 & 28.6 & 29.0 & 36.9 & 36.9 & 19.9 & 21.6 & 132.4 & 132.3 & 0.1 & 26.4 & 27.8 & 34.8 & 34.7 & 13.0 & 17.8 & 89.1 & 87.4 \\
\hline 0.2 & 26.3 & 26.3 & 32.8 & 33.0 & 22.9 & 23.3 & 103.7 & 107.7 & 0.2 & 24.7 & 25.5 & 31.2 & 31.4 & 17.5 & 20.8 & 77.7 & 81.5 \\
\hline 0.3 & 22.7 & 24.6 & 30.0 & 30.5 & 15.2 & 23.4 & 80.9 & 90.4 & 0.3 & 21.7 & 23.8 & 28.5 & 29.2 & 13.1 & 21.0 & 62.6 & 72.7 \\
\hline 0.4 & 18.6 & 23.1 & 27.1 & 28.3 & 8.0 & 22.4 & 56.2 & 73.3 & 0.4 & 18.1 & 22.5 & 25.9 & 27.3 & 7.6 & 20.9 & 46.0 & 64.0 \\
\hline 0.5 & 15.2 & 21.9 & 24.1 & 26.4 & 4.5 & 21.2 & 34.7 & 59.6 & 0.5 & 14.8 & 21.3 & 23.2 & 25.5 & 4.4 & 19.7 & 31.1 & 52.8 \\
\hline 0.6 & 12.4 & 20.8 & 21.0 & 24.7 & 2.8 & 19.6 & 20.3 & 47.7 & 0.6 & 12.0 & 20.2 & 20.1 & 23.7 & 2.8 & 18.5 & 18.2 & 41.7 \\
\hline 0.7 & 10.1 & 19.4 & 17.6 & 22.8 & 2.0 & 16.8 & 11.0 & 36.0 & 0.7 & 9.8 & 19.0 & 17.1 & 22.1 & 2.0 & 16.3 & 10.5 & 33.6 \\
\hline 0.8 & 8.3 & 17.1 & 14.3 & 20.8 & 1.5 & 11.2 & 5.8 & 25.7 & 0.8 & 7.9 & 16.7 & 13.8 & 19.5 & 1.5 & 11.1 & 5.7 & 21.0 \\
\hline 0.9 & 6.9 & 12.1 & 10.4 & 17.8 & 1.2 & 4.0 & 2.7 & 14.6 & 0.9 & 6.5 & 11.7 & 9.8 & 16.1 & 1.2 & 4.0 & 2.6 & 10.9 \\
\hline
\end{tabular}

\section{Steps of algorithm 2 (EMF2)}

Step 1: An array is created according to any given window size. Fig. 1 provides an example for a $3 \times 3$ array.

Step 2: Pixels are sorted from lowest to highest.

Step 3: The window size for the filter $[\mathrm{mn}]$ is

$$
\alpha=m n \text {. }
$$

Step 4: Let processed pixel value be $P_{\mathrm{e}}$ (153 for Fig. 1), and $P_{\mathrm{y}}$ for middle pixel value in Fig. 2, we obtain

$$
\text { If } P_{\mathrm{e}} \geqslant P_{\mathrm{y}}+\alpha \text { or } P_{\mathrm{e}} \leqslant P_{\mathrm{y}}-\alpha \text {, }
$$

then

$$
P_{\mathrm{e}}=P_{\mathrm{y}} \text {. }
$$

\section{RESULTS}

PSNR and IEF test tools are to be used for algorithm results. Images of Lena, Peppers, Cameraman,
Plane, Baboon, Bridge sizing $512 \times 512$ were used. Results were obtained according to $\alpha=2.3$ for EMF1 Filter. As $3 \times 3$ filter size provides the best results for SMF, test results for SMF were noted based on this size. PSNR and IEF results were shown in Table 1, and graphic results in Fig. 3.

The filters added to images such as noise ratio, standard medial filter (SMF), adaptive median filter (AMF), EMF1, the first method we developed, and EMF2, the second method we developed.

\section{DISCUSSION}

This paper offers two novel methods for noise removal in images. Both methods were applied to 6 images with salt-and-pepper noise. All PSNR and IEF results of the methods to which SMF and AMF were applied were better than SMF and AMF methods, especially in the tests where noise levels are high, much better results were obtained. It is safe to anticipate that much better results are 

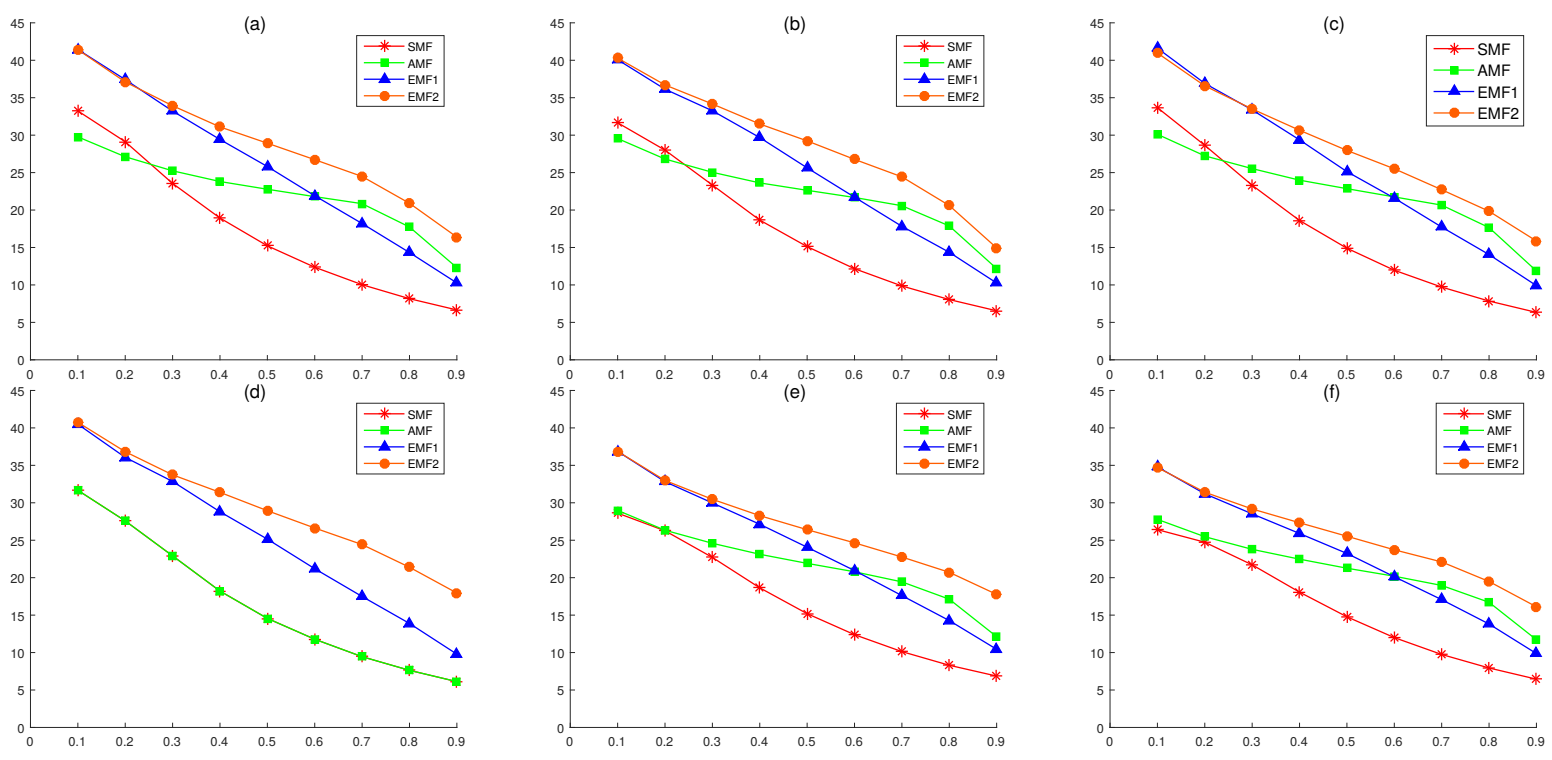

Fig. 3 PSNR Graph, (a) Lena, (b) Peppers, (c) Cameraman, (d) Plane, (e) Baboon, (f) Bridge.
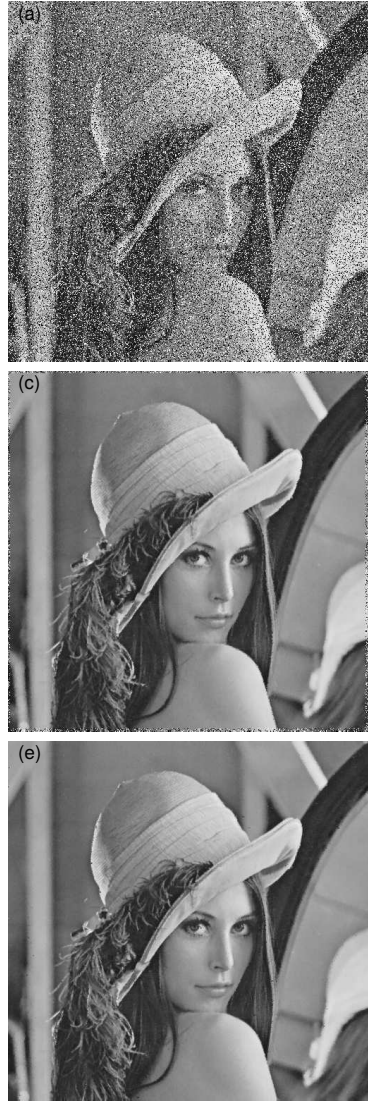

Fig. 4 Lena image quality improvement: (a) 30\% noise added (b) SMF output, (c) AMF output, (d) EMF1 output, (e) EMF2 output, (f) original quality.
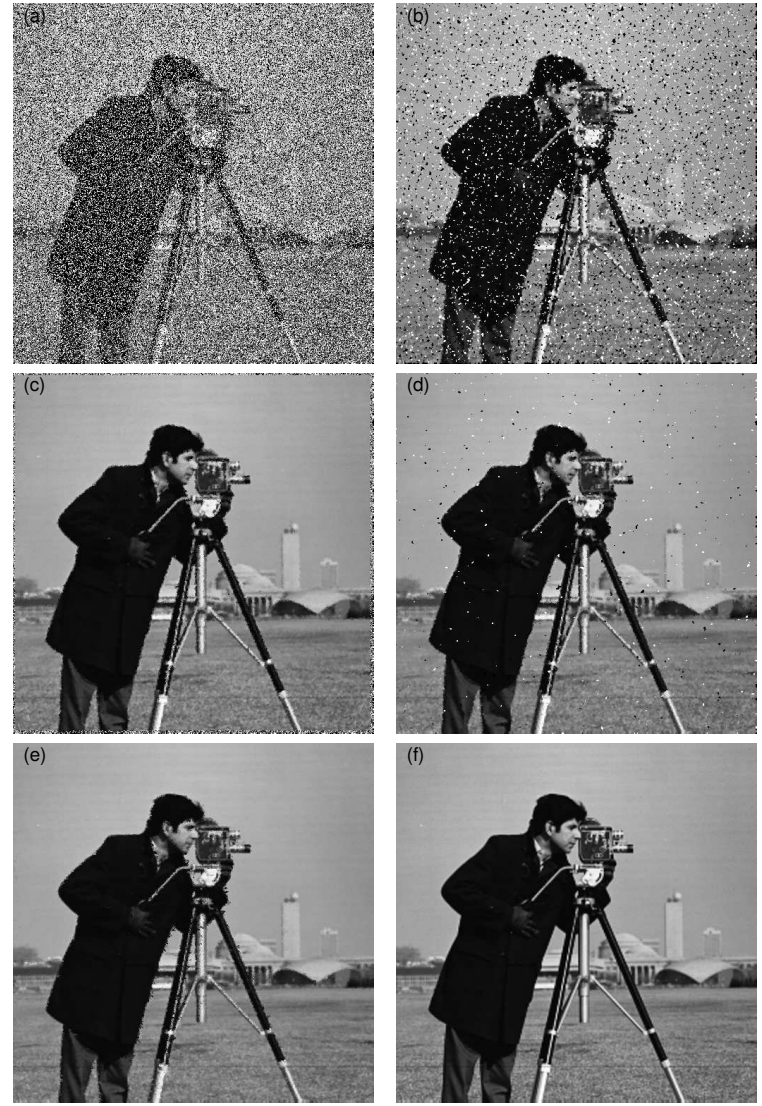

Fig. 5 Cameraman image quality improvement: (a) 50\% noise added, (b) SMF output, (c) AMF output, (d) EMF1 output, (e) EMF2 output, (f) original quality. 

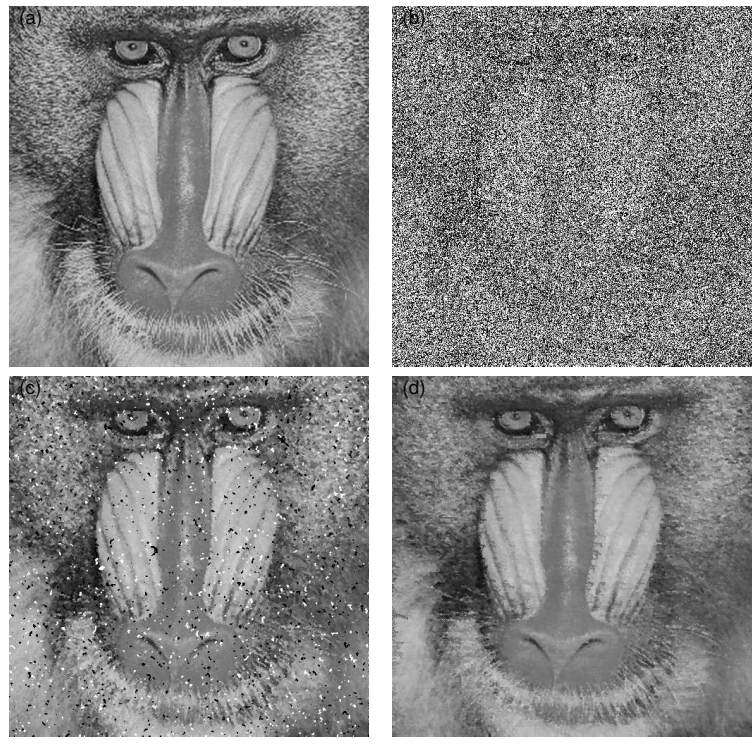

Fig. 6 Baboon image quality improvement, (a) original quality, (b) 70\% noise added, (c) EMF1 output, (d) EMF2 output.

likely to be obtained with the help of classifications of pixel selection in the algorithms and statistical applications. It is anticipated that further research is to be carried out by applying both methods to edge detection algorithm. Additionally, these two novel methods, upon being tested, may also be used in images in special areas. They may produce better results in object recognition and detection applications.

Acknowledgements: The authors express their sincere thanks to the referees and editors for the careful and details reading of the manuscript and very helpful suggestions that improved the manuscript substantially.

\section{REFERENCES}

1. Zhou YY, Ye ZF, Huang JJ (2012) Improved decisionbased detail-preserving variational method for removal of random-valued impulse noise. IET Image Process 6, 976-85.

2. Aiswarya K, Jayaraj V, Ebenezer D (2010) A new and efficient algorithm for the removal of high density salt-and-pepper noise in images and videos. In: Proceedings of the 2nd International Conference on Computer Modeling and Simulation, pp 409-13.

3. Esakkirajan S, Veerakumar T, Subramanyam AN, Chand CHP (2011) Removal of high density salt-andpepper noise through modified decision based unsymmetric trimmed median filter. IEEE Signal Process Lett 18, 287-90.

4. Lu P, Wang W (2011) An efficient switching median filter based on local outlier factor. IEEE Signal Process Lett 18, 551-4.

5. Ng PE, Ma KK (2006) A switching median filter with boundary discriminative noise detection for extremely corrupted images. IEEE Trans Image Process 15, 1506-16.

6. Zhang S, Karim MA (2002) A new impulse detector for switching median filters. IEEE Signal Process Lett 9, 360-3.

7. Toh KKV, Isa NAM (2010) Noise adaptive fuzzy switching median filter for salt-and-pepper noise reduction. IEEE Signal Process Lett 17, 281-4.

8. Toh KKV, Ibrahim H, Mahyuddin MN (2008) Saltand-pepper noise detection and reduction using fuzzy switching median filter. IEEE Trans Consum Electron 54, 1956-61.

9. Van de Ville D, Nachtegael M, Van der Weken D, Kerre EE, Philips W, Lemahieu I (2003) Noise reduction by fuzzy image filtering. IEEE Trans Fuzzy Syst 11, 429-36.

10. Xu H, Zhu C, Peng H, Wang D (2004) Adaptive fuzzy switching filter for images corrupted by impulse noise. Pattern Recogn Lett 25, 1657-63.

11. Nair MS, Raju G (2012) A new fuzzy-based decision algorithm for high-density impulse noise removal. Signal Image Video Process 6, 579-95.

12. Wikipedia (2015) Peak signal-to-noise ratio, http:// en.wikipedia.org/wiki/Peak_signal-to-noise_ratio.

13. Srinivasan KS, Ebenezer D (2007) A new fast and efficient decision-based algorithm for removal of highdensity impulse noises. IEEE Signal Process Lett 14, 189-92.

14. Nasri M, Saryazdi S, Nezamabadi-pour H (2013) A fast adaptive salt and pepper noise reduction method in images. Circ Syst Signal Process 32, 1839-57. 